\title{
Stratégies de requête dans un bureau de tabac et dans son équivalent finlandais $R$-kioski
}

\author{
Tuuli Holttinen \\ Université de Helsinki, Finlande \\ tuuli.holttinen@helsinki.fi
}

\begin{abstract}
Résumé. Cet article examine les façons dont les requêtes sont produites dans deux langues-cultures différentes, le français et le finnois. Plus spécifiquement, l'étude se concentre sur les requêtes verbales et non-verbales dans un bureau de tabac français et dans son équivalent finlandais, R-kioski. Il s'agit donc d'interactions commerciales quotidiennes dans deux magasins où une partie des produits sont en libre-service tandis que d'autres produits doivent être demandés au buraliste. L'objectif de cette étude est de trouver les principales différences existant entre les corpus français et finnois. Les données consistent en des enregistrements authentiques et leurs transcriptions (au total 101 situations de service françaises et 84 situations de service finnoises). Notre méthodologie s'inspire aussi bien de l'analyse conversationnelle que de la pragmatique comparée. Les requêtes verbales sont analysées selon leur stratégie, leur perspective et leur modification interne. L'étude montre que, de manière générale, les stratégies de requête sont assez similaires dans les deux corpus bien que la distribution des éléments étudiés y varie. Dans les deux cas, les requêtes verbales sont plus courantes que les requêtes non-verbales et la stratégie la plus employée est la tournure sans verbe (p. ex. « le Figaro s'il vous plaît »). L'analyse fait émerger trois différences principales : (1) en français, les affirmations d'un désir (p. ex. " je voudrais des enveloppes ») sont assez courantes tandis qu'en finnois cette stratégie n'apparait pas ; (2) en finnois, la perspective impersonnelle est plus employée qu'en français et (3) le français utilise nettement plus de modificateurs internes que le finnois.
\end{abstract}

\begin{abstract}
This paper examines the ways requests are made in two different languages, French and Finnish. More specifically, this study focuses on verbal and non-verbal requests in a French convenience store, bureau de tabac, and the Finnish equivalent, R-kioski. The focus of the study is on everyday interactions in two shops where some of the products are available via self-service whereas other products have to be asked for from the salesperson. The goal is to find the main differences between the French and Finnish data sets, which consist of videotaped service encounters and their transcriptions (101 French and 84 Finnish service encounters). The methodology adopted in this study is inspired by both conversation analysis and cross-cultural pragmatics. Verbal requests are analyzed according to their strategy, perspective, and internal modification. The paper shows that on a general level request strategies are quite similar in both data sets even though the distribution of different elements varies to some extent. In both data sets verbal requests are more common than non-verbal requests, and the most used strategy involves constructions without a verb (e. g. "le Figaro s'il vous plait"). The analysis reveals three main differences: (1) in French, want statements (e. g. "je voudrais des enveloppes") are quite common whereas Finnish does not make use of this strategy; (2) in Finnish, the impersonal perspective is used more often than in French, and (3) internal modifiers are clearly more common in French than in Finnish.
\end{abstract}




\section{Introduction}

Dans la vie quotidienne, tout individu en interaction avec d'autres se voit obligé de réaliser des requêtes. Par conséquent, l'acte de requête a été décrit comme « l'activité la plus fondamentale et la plus répandue de l'interaction sociale » (Drew \& Couper-Kuhlen, $2014: 1$ ). L'un des milieux les plus fertiles pour la production de requêtes est le contexte commercial. En effet, l'acte de requête y est essentiel pour construire les rôles de client et de vendeur (Hmed, 2008 : 260). Les requêtes comme « le Figaro s'il vous plaît » ou «je voudrais des enveloppes » peuvent sembler banales car ce sont des pratiques routinières dont on ne s'aperçoit pas en les utilisant - il est d'ailleurs plus facile de se rendre compte de routines d'une autre culture que la sienne (Traverso, 2006 : 106). En linguistique, c'est le domaine de la pragmatique comparée (cross-cultural pragmatics) qui s'intéresse à révéler systématiquement les différences de pratiques entre deux ou plusieurs langues-cultures.

Les requêtes ont été largement étudiées en partie parce qu'elles ont traditionnellement été considérées comme des Face Threatening Acts (des actes menaçants pour la face) : selon la théorie de politesse de Brown \& Levinson, les requêtes menacent la liberté d'action de l'interlocuteur, c'est-à-dire sa face négative (Brown \& Levinson, 1987 : 65-66). À cause de leur nature menaçante, elles sont fréquemment adoucies de différentes manières linguistiques et paralinguistiques. La façon de formuler une requête dépend fortement du contexte où elle est produite : les paramètres sociaux comme la scène (familière vs. commerciale vs. professionnelle), la relation entre les interlocuteurs (distance sociale et relation hiérarchique) et le «poids » de la requête (imposition rate) ont un grand effet sur sa réalisation (cf. Brown \& Levinson, 1987, Blum-Kulka \& House, 1989, Kerbrat-Orecchioni, 2001a : 105-106). Cependant, l'importance de chaque facteur varie entre les cultures (voir p. ex. Brown \& Levinson, 1987, Blum-Kulka, House \& Kasper, 1989). On pourrait croire que le caractère menaçant des requêtes disparaîtrait en contexte commercial parce que la requête $\mathrm{y}$ est en fait quelque chose d'attendu et d'avantageux pour les deux participants à l'interaction (le client et le vendeur). Pourtant, comme l'affirme Kerbrat-Orecchioni (2001b : 13), « même dans un tel contexte, la requête conserve quelque chose de son caractère « dérangeant », puisque le locuteur éprouve le besoin d'en adoucir la formulation ». Il est ainsi intéressant d'étudier les façons dont les requêtes sont formulées dans le contexte commercial.

L'objectif de cet article est de comparer les différentes réalisations verbales et non-verbales des requêtes françaises et finnoises d'un point de vue pragmalinguistique. La comparaison de ces deux langues-cultures est particulièrement intéressante parce que le finnois et le français représentent deux familles linguistiques différentes (langue indo-européenne vs. langue finno-ougrienne). Plus spécifiquement, nous étudierons les réalisations des requêtes dans un bureau de tabac situé à Lyon et dans son équivalent finlandais, R-kioski, situé à Helsinki. Il s'agit donc d'une étude de cas comparative. La question de recherche à laquelle nous tenterons de répondre est la suivante : quelles sont les similitudes et les différences principales entre les requêtes françaises et finnoises dans un bureau de tabac et dans son équivalent finlandais $R$-kioski?

\section{Corpus et méthodes}

Les données utilisées consistent en des enregistrements et des transcriptions de situations de service authentiques françaises et finlandaises. L'analyse des données authentiques a été caractérisée comme la seule méthode "véritablement fiable » pour une approche comparative (Kerbrat-Orecchioni, 2005a : 290). 
Pour pouvoir mener une analyse interculturelle, il faut vérifier que les interactions se passent dans des conditions comparables : si les sites ne sont pas suffisamment similaires, les différences éventuelles ne peuvent pas être expliquées par la variation culturelle (Kerbrat-Orecchioni, 2005a : 289-290, Traverso, 2006). Le tableau 1 présente les variables extérieures des commerces à comparer dans cet article, le bureau de tabac français et le $R$-kioski finlandais.

\begin{tabular}{|c|c|c|}
\hline & Bureau de tabac & R-kioski \\
\hline Nombre de situations de service ${ }^{I}$ & 101 & 84 \\
\hline Variété de produits (achetés) & $\begin{array}{l}\text { Journaux/magazines, tabac, } \\
\text { billets de loterie, produits } \\
\text { alimentaires, autres produits (p. } \\
\text { ex. timbres, allumettes), } \\
\text { vignettes de voiture }\end{array}$ & $\begin{array}{l}\text { Journaux/magazines, tabac, } \\
\text { billets de loterie, produits } \\
\text { alimentaires, autres produits } \\
\text { (p. ex. timbres, allumettes), } \\
\text { tickets de bus }\end{array}$ \\
\hline $\begin{array}{l}\text { Organisation physique des } \\
\text { produits }\end{array}$ & $\begin{array}{l}\text { Libre-service }+ \text { une partie des } \\
\text { produits derrière le comptoir }\end{array}$ & $\begin{array}{l}\text { Libre-service + une partie des } \\
\text { produits derrière le comptoir }\end{array}$ \\
\hline Buraliste & Femme, $35-45$ ans & Femme, environ 40 ans \\
\hline Emplacement du magasin & Grande ville (Lyon) & Grande ville (Helsinki) \\
\hline Année d'enregistrement & 1987 & 2002 \\
\hline
\end{tabular}

Tableau 1. Variables extérieures des corpus

Le corpus français est composé de 101 situations de service dans un bureau de tabac lyonnais, tandis que les 84 situations finlandaises ont lieu dans un R-kioski à Helsinki. Les deux corpus se correspondent en ce qui concerne l'emplacement du magasin, l'âge et le sexe des buralistes ainsi que l'organisation physique des produits. Cette dernière variable est importante car elle a une grande influence sur la construction des interactions commerciales (cf. Traverso, 2006). Quant aux produits vendus, les deux commerces sont également comparables même s'il y existe quelques différences. Les corpus présentent néanmoins le désavantage de ne pas avoir été enregistrés à la même période et de ne pas être tout à fait récents $^{2}$. Il faut donc tenir compte du fait que nous ne pouvons pas en tirer des conclusions applicables directement aux interactions d'aujourd'hui. Les stratégies de requête utilisées dans ces commerces sont cependant fortement ritualisées (voir Aijmer, 1996) si bien qu'elles ne sont pas aussi aptes aux changements dans le temps que le lexique par exemple. Ainsi, malgré ces quelques différences et étant donné que la plupart des variables extérieures se correspondent, considérons-nous les deux corpus comme comparables. En outre, il serait quasiment impossible de trouver deux ensembles qui se correspondent parfaitement.

En ce qui concerne les méthodes, nous nous inspirons aussi bien de l'analyse conversationnelle que de la pragmatique comparée. L'analyse conversationnelle s'intéresse surtout à l'organisation des séquences (voir p. ex. Schegloff, 2007). Dernièrement, les analyses conversationnelles sur la requête se sont également concentrées sur la multimodalité des actions (voir Drew \& Couper-Kuhlen (éds), 2014). Nous tiendrons compte de ces points de vue notamment dans la présentation des requêtes (partie 3), mais nous nous appuierons également sur des outils d'analyse exploités en pragmatique comparée. Ces outils se fondent surtout sur le travail du groupe CCSARP (Cross-Cultural Speech Act Realization Project, BlumKulka, House \& Kasper, 1989). Suivant la tradition de ce domaine, notre analyse se concentrera sur les manières dont les requêtes verbales sont formulées. Plus précisément, nous analyserons les actes principaux des requêtes du point de vue de leur stratégie, de leur perspective et de leur modification interne. L'objectif de la présente analyse est de comparer la manière dont ces éléments sont distribués dans les deux corpus (partie 4). La combinaison des deux traditions, l'analyse conversationnelle et la pragmatique comparée, nous permettra d'analyser les données d'une façon variée et étendue. 


\section{Présentation des requêtes}

\subsection{Principes d'analyse}

En situation de service, la requête est généralement située au début de l'interaction, après les salutations ${ }^{3}$ (voir p. ex. Traverso, 2006, Lappalainen, 2009a). Bien que toutes les parties de l'échange constituent des objets d'étude intéressants, nous ne nous concentrerons que sur la deuxième partie de ces interactions, c'est-à-dire sur la séquence de requête. Comme le font remarquer plusieurs chercheurs (p. ex. Drew \& Couper-Kuhlen, 2014), les requêtes peuvent être réalisées d'une façon verbale ou non-verbale. Les deux modalités sont utilisées dans les petits commerces, parfois conjointement :

Exemple 1. TABAC CL10 ${ }^{4}$ (CL10 = homme, env. 25 ans)

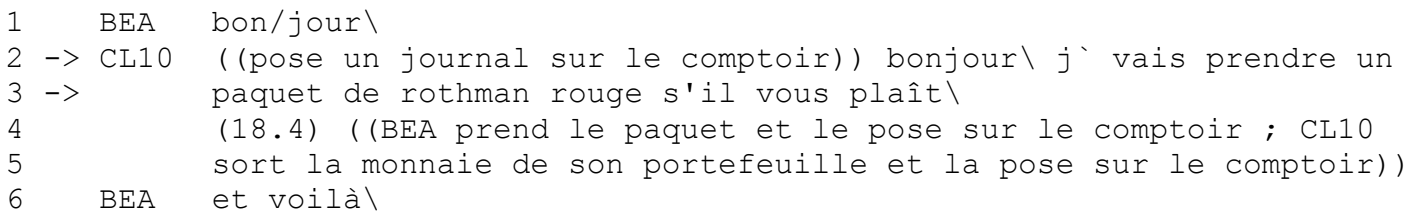

Dans cet exemple, le client pose un journal sur le comptoir, ce qui correspond à une requête non-verbale, et formule en même temps une requête verbale « je vais prendre un paquet de rothman rouge s'il vous plaît ». Il y a donc deux actes de requête distincts, produits à l'aide des deux modalités.

Dans les situations analysées, la plupart des requêtes sont verbalisées (voir tableau 2 ci-après). Il y a une légère différence entre les données : les requêtes non-verbales sont un peu plus communes dans le corpus français que dans le corpus finnois. Cette différence peut être liée au fait que les clients français achètent plus de produits en libre-service (surtout des journaux et des magazines) que les clients finlandais.

\begin{tabular}{|l|c|c|c|}
\hline & & Bureau de tabac & R-kioski \\
\hline Requêtes non-verbales & $\mathrm{n}$ & 41 & 23 \\
\hline Requêtes verbales & $\boldsymbol{\%}$ & $\mathbf{3 1 , 5}$ & $\mathbf{2 1 , 7}$ \\
\hline & $\mathrm{n}$ & 89 & 83 \\
\hline Total & $\boldsymbol{\%}$ & $\mathbf{6 8 , 5}$ & $\mathbf{7 8 , 3}$ \\
\hline
\end{tabular}

Tableau 2. Distribution des requêtes produites dans le bureau de tabac et le R-kioski

Nous avons tenu compte de tous les actes de requête principaux (Blum-Kulka, House \& Kasper, 1989 : 275) qui se trouvent dans une seule interaction : si le client achète un paquet de cigarettes et une boîte d'allumettes et le fait en deux requêtes séparées (autrement dit en deux actes principaux), nous considérons qu'il y a deux requêtes distinctes (voir l'exemple 2).

Exemple 2. TABAC CL17 (CL17 = homme, env. 40 ans)

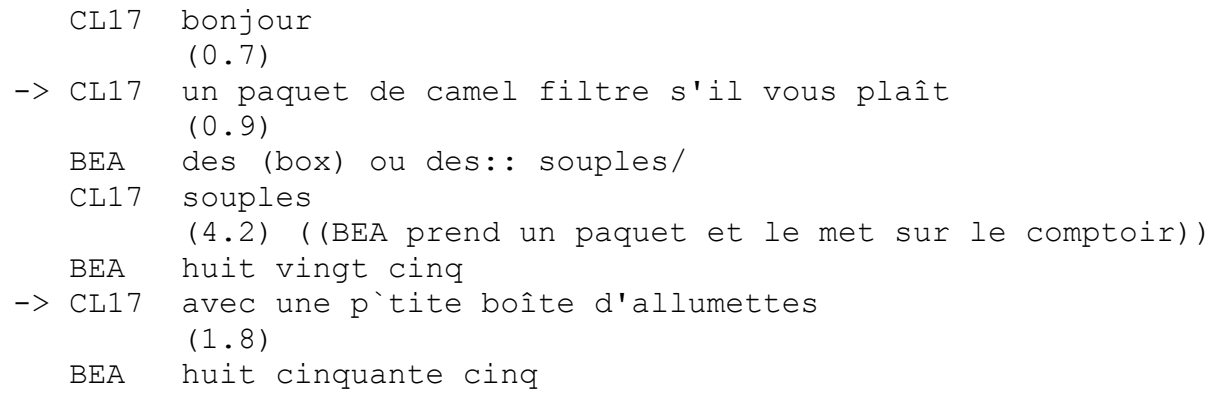


Dans cet exemple, le client produit sa première requête (ligne 3), après quoi la buraliste demande quel type de paquet il veut (ligne 5). Après la réponse du client (ligne 6), la buraliste accomplit la requête et annonce le prix (lignes 7 et 8 ). Cela est pourtant suivi d'une deuxième requête : « avec une p`tite boîte d'allumettes » (ligne 9). Il y a donc deux requêtes distinctes dans cette interaction. Notons que la formulation de la seconde requête montre qu'il s'agit d'une requête de suivi : la première requête ne pourrait pas être formulée en utilisant la préposition « avec ».

Il faut cependant noter qu'il existe une différence entre les deux corpus en ce qui concerne les interactions où plusieurs requêtes sont produites : dans le corpus finnois, la buraliste a tendance à demander automatiquement si le client désire autre chose tandis que la buraliste française ne le fait pas une seule fois. Il s'agit probablement d'une différence entre les habitudes des buralistes (plutôt que d'une différence entre les langues-cultures). Voici un exemple finnois où la vendeuse pose la question «avec ceci ? à laquelle la cliente répond par une deuxième requête :

Exemple 3. R-KIOSKI T6025 ( $\mathrm{A}=$ femme, env. 65 ans)

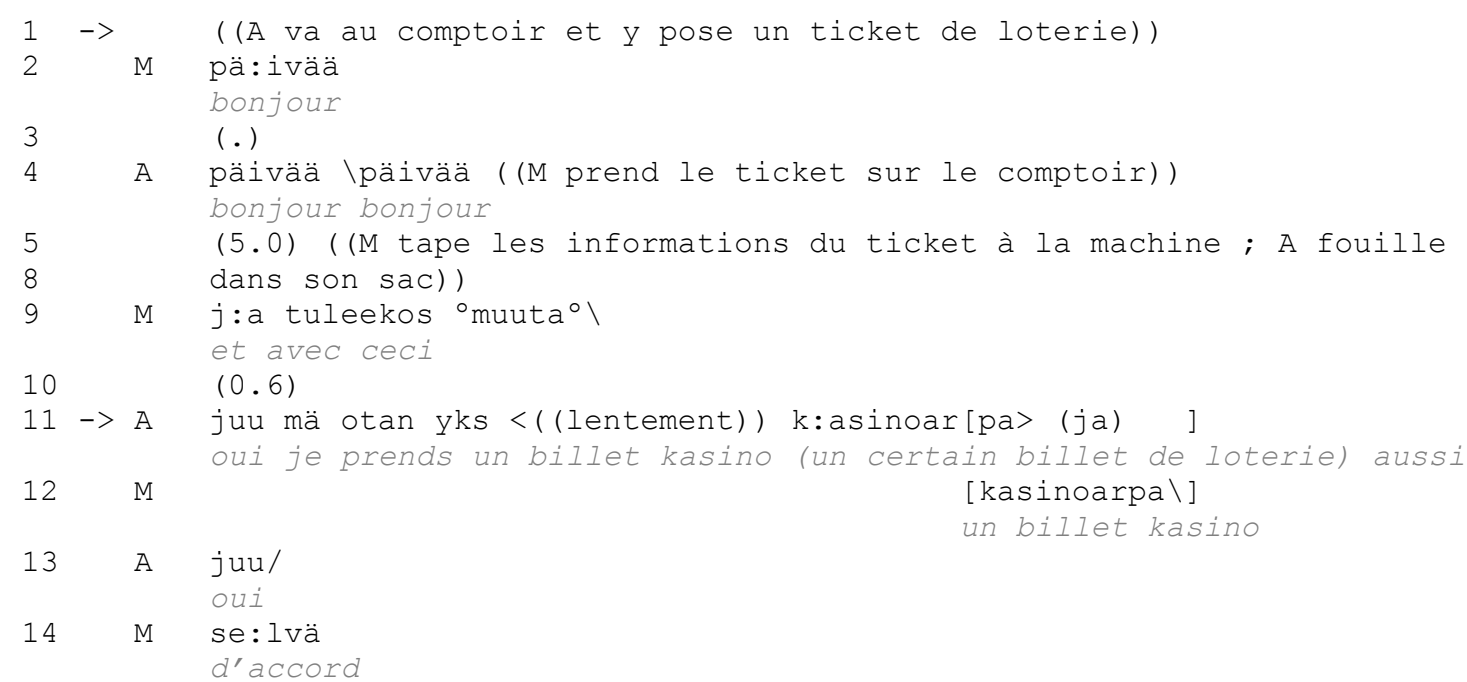

Les requêtes en deuxième position (qui suivent la question «avec ceci ? ») diffèrent un peu des requêtes en première position. Dans l'exemple 3, la cliente commence sa deuxième requête (ligne 11) en répondant « oui » à la question de la buraliste, posée à la ligne 9 - il s'agit donc d'une paire adjacente questionréponse. Le nombre de requêtes finnoises formulées après la question « avec ceci ? » n'est pourtant pas très élevé $(n=6)$ parce que, le plus souvent, le client ne veut pas autre chose. Nous n'avons pas exclu ces requêtes du corpus finnois bien que les séquences où elles se trouvent soient construites légèrement différemment des requêtes qui se trouvent au début de l'interaction.

\subsection{Requêtes non-verbales}

Dans les deux corpus, les requêtes non-verbales sont courantes puisque les commerces étudiés contiennent des produits en libre-service. Pourtant, les requêtes non-verbales sont un peu plus fréquentes dans le corpus français (32\% des requêtes) que dans le corpus finnois ( $22 \%$ des requêtes) (voir tableau 2 ci-dessus). Voici deux exemples de requêtes non-verbales typiques pour les données étudiées :

Exemple 4. TABAC CL29 (CL29 = femme, env. 55 ans)

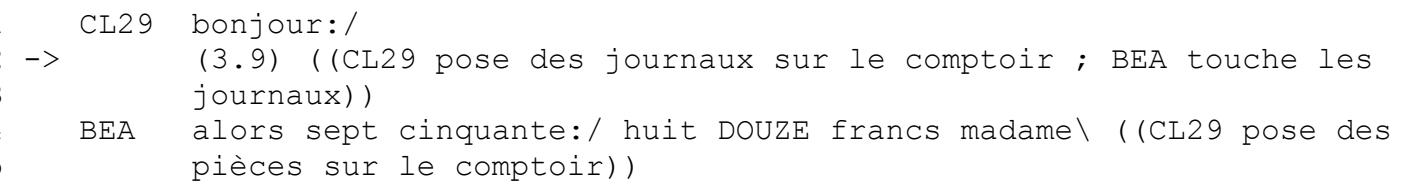




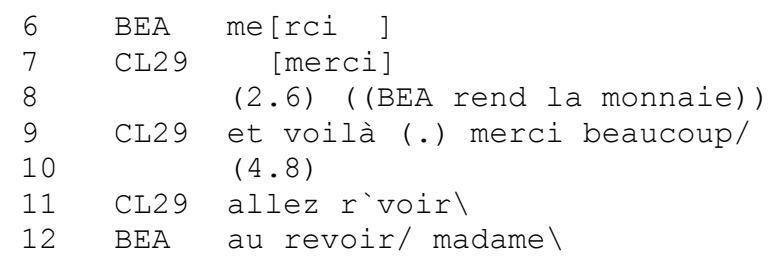

Exemple 5. R-KIOSKI T510 (A = femme, env. 25 ans)

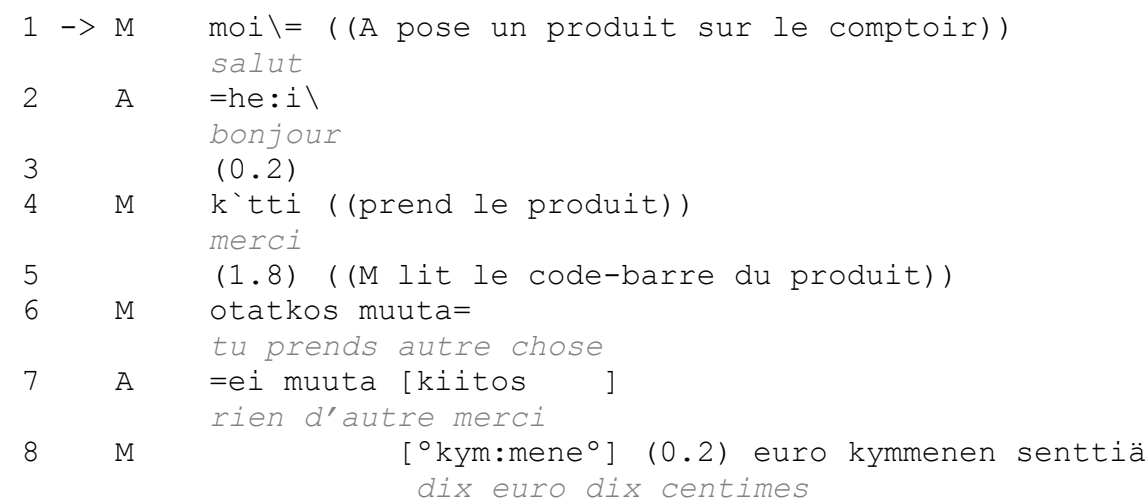

Dans ces exemples, la cliente va à la caisse, salue la buraliste et pose des objets sur le comptoir, ce que la buraliste interprète comme une requête de facturer les produits. Notons que la buraliste française annonce le prix immédiatement après la requête non-verbale tandis que la buraliste finlandaise demande si le client veut autre chose.

Rossi (2014) a étudié les requêtes non-verbales dans l'interaction quotidienne. Il affirme qu'elles sont utilisées pour épargner aussi bien les efforts de la personne qui produit la requête (requester) que ceux de la personne à qui la requête est adressée (requestee) :

\begin{abstract}
«If a nonverbal form is enough to achieve understanding, the requester should not add extra cost by accompanying it with unnecessary language. Adding another semiotic layer implies more effort both for the requester to produce and for the requestee to process. » (Rossi, 2014 : 328-329)
\end{abstract}

Cela semble être également vrai dans le cas des situations de service étudiées ici, où le nom du produit n'est prononcé que rarement, quand il est nécessaire.

En parlant des requêtes non-verbales et de la multimodalité qui y est liée, il faut noter que, dans le corpus français, qui est plus ancien que le corpus finnois, le client n'est pas obligé de poser l'objet sur le comptoir mais il suffit que la buraliste voie l'objet en question pour pouvoir l'encaisser. Dans le corpus finnois, par contre, le client se voit bien plus souvent obligé de faire un effort physique et de mettre l'objet sur le comptoir car la buraliste utilise un lecteur électronique pour lire le code-barre du produit.

Même si la requête n'est pas toujours verbalisée, cela ne veut pas dire que les clients ne disent rien du tout : ils peuvent parler d'autres choses et produire la requête de manière non-verbale.

Exemple 6. TABAC CL71 (CL71 = homme, env. 70 ans)

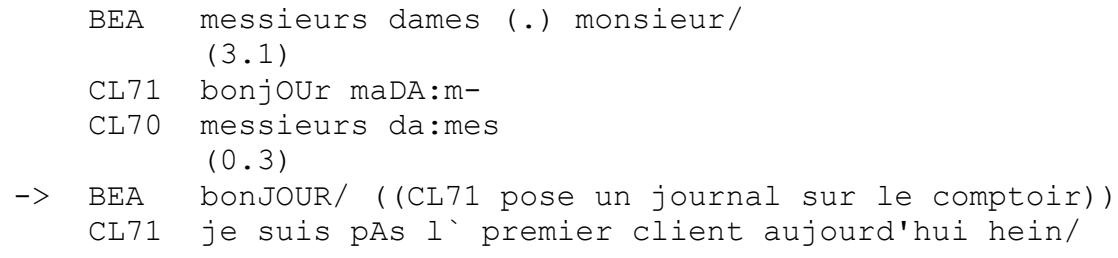




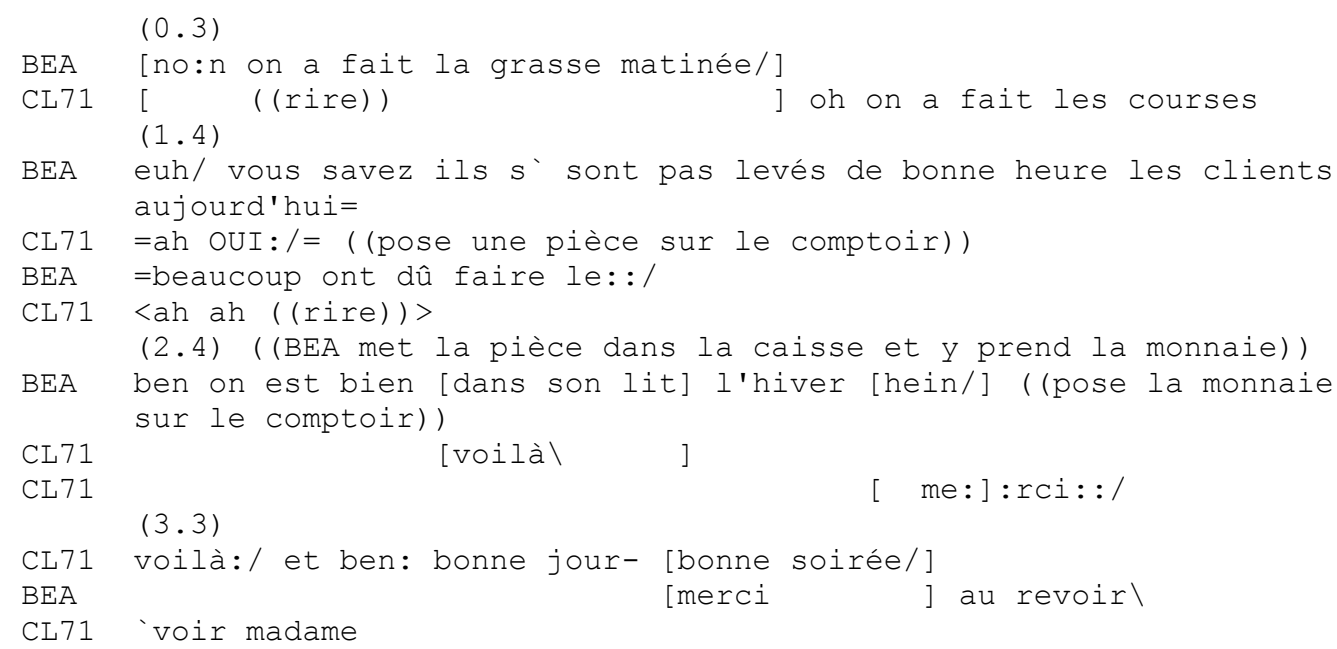

Dans cet exemple, la requête est réalisée non-verbalement (ligne 6). Ici, la production verbale n'est pas liée à la transaction; elle sert plutôt à promouvoir les relations interpersonnelles entre les locuteurs. Cela révèle le caractère semi-institutionnel des interactions en site commercial (KerbratOrecchioni \& Traverso, $2008: 8$ ) : d'une part, il s'agit d'interactions formelles et routinières dont tout le monde connaît le script et d'autre part, il peut également s'y dérouler de « vraies » conversations dont la fonction n'est pas transactionnelle mais plutôt sociale.

\subsection{Requêtes verbales}

Comme le montre le tableau 2 (voir ci-dessus), les interactions où la requête n'est pas du tout verbalisée sont assez courantes dans les deux corpus. La requête est cependant verbalisée dans la plupart des interactions (69\% des requêtes françaises et $78 \%$ des requêtes finnoises). Nous venons de mentionner que les requêtes non-verbales sont utilisées lorsque le produit demandé se trouve en libre-service. Toutes les requêtes verbales ne sont cependant pas liées aux produits qui se trouvent derrière le comptoir :

Exemple 7. TABAC CL94 (CL94 = homme, env. 50 ans)

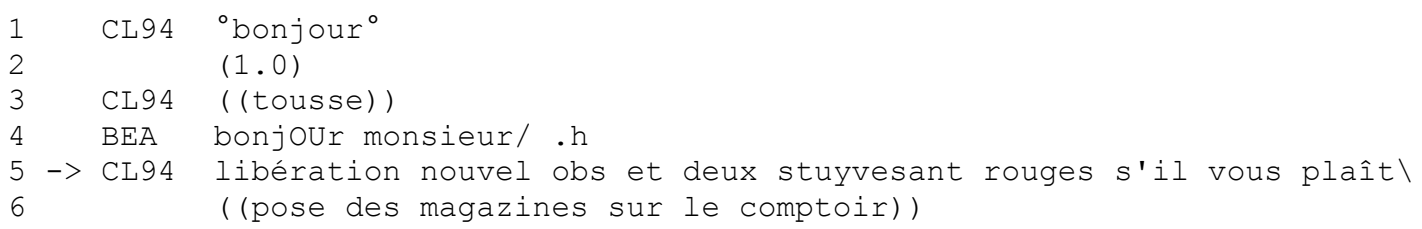

Exemple 8. R-KIOSKI T603 (A = homme, env. 65 ans)

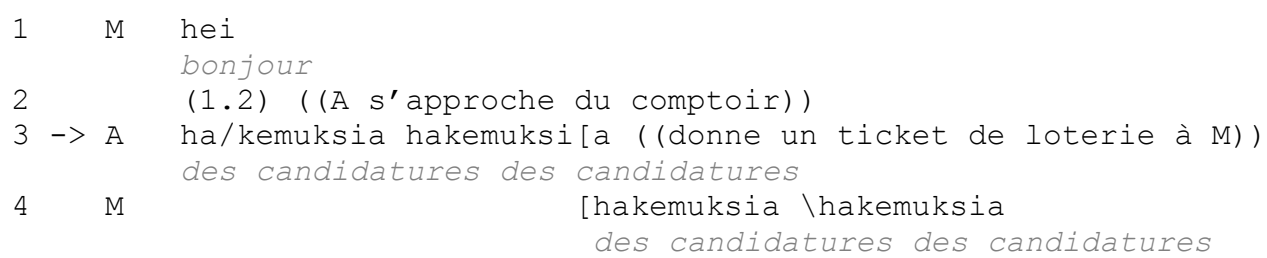

Les requêtes comme celle de l'exemple 7 (ligne 5) sont assez courantes dans les deux corpus : quand un client achète un produit en libre-service et un produit qui se trouve derrière le comptoir, il produit souvent une requête verbale dans laquelle il mentionne les deux produits. Dans l'exemple 8, par contre, le client ne précise pas ce qu'il est en train d'acheter (un certain ticket de loterie) mais dit «hakemuksia hakemuksia » («des candidatures des candidatures »). Le client profite ainsi de la fonction ludique du 
langage : en réalité, il n'est pas en train de soumettre une candidature mais de faire un achat. Le langage ludique est d'ailleurs fréquent dans les interactions en $R$-kioski, surtout quand il s'agit d'acheter un ticket de loterie (voir en détail chez Haakana \& Sorjonen, 2009).

\subsubsection{Stratégie de requête}

Les stratégies de requête utilisées dans les deux corpus se classent dans les catégories suivantes :

\begin{tabular}{|c|c|c|}
\hline Stratégie & Exemple français & Exemple finnois \\
\hline Tournure sans verbe & «X », «X s'il vous plaît » & «X $», \ll X$ kiitos » \\
\hline Impératif & «donnez-moi X » & «laita X» \\
\hline $\begin{array}{l}\text { Affirmation avec le verbe } \\
\text { «acheter» }\end{array}$ & « je vais acheter $X »$ & « ostaisin $X »$ \\
\hline $\begin{array}{l}\text { Affirmation avec le verbe } \\
\text { «prendre» }\end{array}$ & $\begin{array}{l}\text { «je prends } X », \ll \text { je vais prendre } \\
X »\end{array}$ & $\ll \operatorname{otan} X »$ \\
\hline Affirmation d'un désir & «je voudrais $X »$ & $\begin{array}{l}\text { « haluaisin } X » \text { (non attesté dans } \\
\text { le corpus) }\end{array}$ \\
\hline Autres affirmations & $\begin{array}{l}\text { «je cherche } X », \text { « vous me } \\
\text { mettez } X »\end{array}$ & « laitetaan X » \\
\hline $\begin{array}{l}\text { Question sur la } \\
\text { disponibilité }\end{array}$ & $\begin{array}{l}\text { «est-ce que vous avez } X », \text { est- } \\
\text { ce que vous vendez } X »\end{array}$ & $\begin{array}{l}\text { «saako tästä } X \text { », « onko } \\
\text { teillä/sinulla X » }\end{array}$ \\
\hline Question de prédiction & «vous me donnez $X »$ & «annatko X », « saanko X » \\
\hline $\begin{array}{l}\text { Question sur une condition } \\
\text { de réussite }\end{array}$ & $\begin{array}{l}\text { « est-ce que je pourrais avoir/voir } \\
X »\end{array}$ & $\begin{array}{l}\text { « saatko laitettua } X \text { », « voitko } \\
\text { antaa } X »\end{array}$ \\
\hline Autres questions & $\begin{array}{l}\text { «X se trouve où », « ça c'est } \\
\text { combien » }\end{array}$ & « mitä tämä maksaa » \\
\hline
\end{tabular}

Tableau 3. Stratégies de requête

Le tableau 3 présente les stratégies de requête ainsi que des exemples prototypiques pour chaque catégorie. Cependant, d'autres formulations sont également possibles : ces exemples montrent seulement «l'idée fondamentale » de chaque stratégie. De plus, les exemples ont été légèrement simplifiés pour le tableau même s'ils ont été créés à partir des données.

Les stratégies listées ici correspondent en partie à des stratégies ou à des classifications utilisées par d'autres chercheurs. Par exemple, la stratégie que nous désignons par «tournure sans verbe ${ }^{6}$ a également été utilisée par Kerbrat-Orecchioni (2001b), mais elle la nomme « tournure elliptique ». Nous préférons ne pas utiliser le terme «ellipse ». Comme le fait remarquer Kerbrat-Orecchioni elle-même (2001b : 10), «l'interprétation [de ces tournures] pose d'ailleurs problème : faut-il supposer élidé un «Donnez-moi...», un «Je veux... » ou un «Je voudrais...»? ». En ce qui nous concerne, nous ne présupposons pas l'absence d'un élément qui aurait subit une ellipse mais partons du principe que ces tournures forment des requêtes telles quelles.

Une catégorisation des stratégies de requête couramment utilisée dans la recherche est celle créée par Blum-Kulka, House \& Kasper (1989). Elles classent les requêtes du type «X, s'il vous plaît » et «donnez-moi X » dans la même catégorie (mood derivable). Nous voulons cependant établir une distinction entre les tournures sans verbe et les impératifs pour obtenir une classification suffisamment fine. Sorjonen, Raevaara \& Lappalainen (2009), elles aussi, font cette distinction, même si leur classification des requêtes verbales n'est pas aussi détaillée que celle utilisée dans cette analyse.

La classification de Blum-Kulka, House \& Kasper (1989) présente un continuum allant des stratégies directes aux stratégies indirectes. Par exemple, les auteurs considèrent les affirmations d'un désir (want 
statements) comme des stratégies directes et les questions sur une condition de réussite (preparatory questions) comme des stratégies indirectes. Cette classification a pourtant été critiquée :

\begin{abstract}
«The authors of the Coding Manual claim that this scheme was guided by the level of indirectness of the requests. The criteria for indirectness, however, remain implicit or seem to emerge from the imposed hierarchy of the listed classes. This way the proceeding runs the risk of resulting in circular reasoning. » (Van Mulken, $1996: 692)$
\end{abstract}

Ici, nous ne prétendons pas que les différentes stratégies suivent un tel continuum : nous avons créé les catégories à partir des caractéristiques syntaxiques et sémantiques des requêtes plutôt que selon leur «level of (in)directness », même si nous admettons que l'utilisation de l'impératif est évidemment plus directe (et ainsi plus menaçante, au moins dans certains contextes et cultures) que l'utilisation d'une question qui laisse (au moins théoriquement) à l'auditeur la possibilité de choisir s'il va accomplir la requête ou pas. En outre, les chercheurs ne sont pas d'accord sur ce qu'il faut considérer comme des stratégies directes et indirectes. Par exemple, Kerbrat-Orecchioni (2001b : 10) considère l'affirmation d'un désir comme une stratégie indirecte, contrairement à Blum-Kulka, House \& Kasper (1989).

Dans cette analyse, nous ne faisons pas de distinction entre les pré-requêtes et les requêtes proprement dites même si cette distinction est souvent faite dans l'analyse conversationnelle traditionnelle (voir par exemple Levinson, 1983). Toutefois, Fox (2015), parmi d'autres, affirme que les questions sur la disponibilité comme «do you have $X$ » ou «can I get $X$ » dans des situations de service ne devraient pas être analysées comme des pré-requêtes. La discussion sur la nature des pré-requêtes est intéressante mais comme ce n'est pas l'objet principal de cette étude, nous nous contentons de dire que nous avons inclus aussi bien les requêtes en «première position », traditionnellement considérées comme des pré-requêtes, que les requêtes en « troisième position » (Levinson, 1983 : 357) dans la classification des stratégies.

\title{
3.3.2 Perspective de requête
}

La perspective de requête est liée à l'agentivité : une requête peut s'orienter vers le locuteur (speakeroriented request) ou l'auditeur (hearer-oriented request). Il est également possible de faire disparaître l'agent soit en s'orientant et vers le locuteur et vers l'auditeur (inclusive request), soit en utilisant une construction impersonnelle (impersonal request). En voici quelques exemples, créés à partir des données :

\begin{tabular}{|c|c|c|}
\hline Perspective & Exemple français & Exemple finnois \\
\hline Orientée vers le locuteur & $\begin{array}{l}\text { «je voudrais } X », \text { je vais } \\
\text { acheter } X »\end{array}$ & «otan $X »$, ostaisin $X »$ \\
\hline Orientée vers l'auditeur & $\begin{array}{l}\text { «donnez-moi } X », \text { « est-ce que } \\
\text { vous vendez } X »\end{array}$ & « laita $X », \ll$ onko sinulla $X »$ \\
\hline Impersonnelle & $\begin{array}{l}\text { «X », «X s'il vous plaît », } \\
\text { «c'est pour } X »\end{array}$ & $\begin{array}{l}\text { «X », «X kiitos », « saako tästä } \\
X », \text { laitetaan } X^{7} »\end{array}$ \\
\hline Inclusive & $\begin{array}{l}\text { « est-ce que nous pouvons } X \text { » } \\
\text { (non attesté dans le corpus) }\end{array}$ & $\begin{array}{l}\text { « voimmeko X » (non attesté } \\
\text { dans le corpus) }\end{array}$ \\
\hline
\end{tabular}

Tableau 4. Perspectives de requête

Cette classification a été utilisée dans la littérature sur la requête dès la publication de Blum-Kulka, House \& Kasper (1989). Blum-Kulka (1989: 59) note que les langues peuvent préférer différentes perspectives en général ou parmi certaines stratégies. Elle fait également remarquer ( $i b i d$.) que le choix de la perspective peut réduire la nature intrusive de la requête : si l'auditeur n'est pas mentionné comme agent, la requête peut sembler moins menaçante.

Dans le «Coding Manuel» de Blum-Kulka, House \& Kasper (1989 : 278), la perspective impersonnelle contient des tournures sans verbe (p. ex. « un peu de silence s'il vous plaît ») ainsi que l'utilisation des agents neutres (p. ex. «Can one ask for a little quiet? »). Toutefois, dans l'étude de Blum-Kulka (1989), seules les stratégies indirectes conventionnelles (c'est-à-dire les questions sur une condition de réussite) 
sont analysées du point de vue de leur perspective. Peterson (2010), quant à elle, a analysé la perspective des requêtes qui contiennent un prédicat. Dans cette étude, nous analysons cependant la perspective de tous les types de requêtes pour avoir une idée générale des choix faits dans ce domaine. Néanmoins, il est vrai que seules les requêtes interrogatives laissent un vrai choix entre les différentes perspectives : il est possible de choisir entre « est-ce que vous pouvez me donner un paquet de cigarettes » et « est-ce que je peux avoir un paquet de cigarettes », alors que l'impératif est toujours orienté vers l'auditeur et une tournure sans verbe est toujours impersonnelle.

\subsubsection{Modification interne}

Une manière d'adoucir la requête, c'est-à-dire de diminuer son caractère intrusif, est de la modifier par des moyens syntaxiques et lexicaux. Blum-Kulka, House \& Kasper (1989 : 281-283) listent différents types de modification interne ; nous nous servirons seulement d'une partie d'entre eux pour deux raisons. Premièrement, dans les corpus commerciaux qui nous concernent, tous les types de modification n'apparaissent pas et deuxièmement, nous avons traité les interrogatives comme des stratégies séparées (et non comme une modification interne). De plus, l'emploi du subjonctif n'est pas optionnel en français et c'est pourquoi ce mode n'est pas considéré comme un modificateur. Nos choix correspondent à ceux de Van Mulken (1996 : 697). Ainsi, la modification interne dans cette étude contient les éléments suivants :

\begin{tabular}{|c|c|c|c|}
\hline & & Exemple français & Exemple finnois \\
\hline \multirow{3}{*}{$\begin{array}{l}\text { Modification } \\
\text { morpho- } \\
\text { syntaxique }\end{array}$} & Conditionnel & «je voudrais $X »$ & $\ll$ ottaisin $X »$ \\
\hline & Imparfait & «je voulais $X »$ & $\begin{array}{l}\text { « halusin } \mathrm{X} »(\text { non } \\
\text { attesté) }\end{array}$ \\
\hline & Négation & «vous n'avez pas de $X »$ & « ei teillä ole $X »$ \\
\hline \multirow{2}{*}{$\begin{array}{l}\text { Modification } \\
\text { lexicale }\end{array}$} & Marqueur de politesse & «X s’il vous plaît » & «X kiitos » \\
\hline & Minimisation & $\begin{array}{l}\text { «je prends un(e) petit(e) } \\
X »\end{array}$ & $\begin{array}{l}\text { « saanko vähän } \mathrm{X} \text { » } \\
\text { (non attesté) }\end{array}$ \\
\hline
\end{tabular}

Tableau 5. Types de modification interne

D'après des études antérieures (Yli-Vakkuri, 2005, Peterson, 2008), le finnois préfère la modification morpho-syntaxique à la modification lexicale pour exprimer la politesse, bien que les deux types de modification soient possibles en finnois. En effet, kiitos ne correspond pas tout à fait aux marqueurs de politesse typiques pour les langues européennes ( $s$ 'il vous plaît français, please anglais, por favor espagnol), mais différents éléments finnois (p. ex. kiitos, olelolkaa hyvä, l'emploi du conditionnel, l'emprunt pragmatique pliis, l'enclitique -han/-hän) peuvent y correspondre (Peterson, 2008, Peterson \& Vaattovaara, 2014). Dans le cadre de notre analyse, kiitos est pourtant le seul élément apparaissant dans le corpus qui puisse être considéré comme un marqueur de politesse.

\section{Formulation des requêtes verbales : résultats globaux}

\subsection{Distribution des stratégies de requête}

La classification des requêtes verbales par leur stratégie donne les résultats suivants : 


\begin{tabular}{|c|c|c|c|}
\hline Stratégie & & Bureau de tabac & R-kioski \\
\hline \multirow{2}{*}{ Tournure sans verbe } & $\mathrm{n}$ & 44 & 49 \\
\hline & $\%$ & 49,4 & $\mathbf{5 9 , 0}$ \\
\hline \multirow[t]{2}{*}{ Impératif } & $\mathrm{n}$ & 2 & 1 \\
\hline & $\%$ & 2,2 & 1,2 \\
\hline \multirow[t]{2}{*}{ Affirmation avec le verbe « acheter » } & $\mathrm{n}$ & 1 & 2 \\
\hline & $\%$ & 1,1 & 2,4 \\
\hline \multirow{2}{*}{ Affirmation avec le verbe « prendre»" } & $\mathrm{n}$ & 8 & 13 \\
\hline & $\%$ & 9,0 & 15,7 \\
\hline \multirow[t]{2}{*}{ Affirmation d'un désir } & $\mathrm{n}$ & 17 & 0 \\
\hline & $\%$ & 19,1 & $\mathbf{0 , 0}$ \\
\hline \multirow[t]{2}{*}{ Autres affirmations } & $\mathrm{n}$ & 5 & 4 \\
\hline & $\%$ & 5,6 & 4,8 \\
\hline \multirow[t]{2}{*}{ Question sur la disponibilité } & $\mathrm{n}$ & 8 & 9 \\
\hline & $\%$ & 9,0 & 10,8 \\
\hline \multirow[t]{2}{*}{ Question de prédiction } & $\mathrm{n}$ & 1 & 2 \\
\hline & $\%$ & 1,1 & 2,4 \\
\hline \multirow[t]{2}{*}{ Question sur une condition de réussite } & $\mathrm{n}$ & 1 & 1 \\
\hline & $\%$ & 1,1 & 1,2 \\
\hline \multirow[t]{2}{*}{ Autres questions } & $\mathrm{n}$ & 2 & 2 \\
\hline & $\%$ & 2,2 & 2,4 \\
\hline Total & $\mathrm{n}$ & 89 & 83 \\
\hline
\end{tabular}

Tableau 6. Distribution des requêtes verbales selon la stratégie

Le tableau 6 montre que la stratégie la plus courante dans les deux corpus est la tournure sans verbe. Cela est bien compréhensible parce que les interactions dans les bureaux de tabac et dans les R-kioski sont souvent courtes et rapides. Selon Kerbrat-Orecchioni (2005b : 92-93), la fréquence de ce type de requête peut être liée justement à la nature des interactions : les clients doivent faire la queue, il s'agit de produits quotidiens et les clients savent ce qu'ils veulent. Elle explique également que l'utilisation d'une telle stratégie pourrait être liée à la politesse parce qu'elle économise le temps du vendeur et d'autres clients. Il y a pourtant une légère différence entre les interactions françaises et finnoises : environ $49 \%$ des requêtes verbales françaises consistent en une tournure sans verbe tandis que le taux correspondant pour le finnois est d'environ $59 \%$. La deuxième stratégie la plus utilisée pour le français est l'affirmation d'un désir $(19 \%)$ tandis que cette stratégie n'est pas utilisée en finnois. Cette observation correspond aux résultats de l'étude de Muikku-Werner (1997) où cette stratégie n'apparaissait guère ${ }^{8}$.

Pour synthétiser les résultats, nous avons regroupé ci-dessous les stratégies en quatre grandes catégories selon le type de proposition utilisé :

\begin{tabular}{|l|c|c|c|}
\hline Stratégie & & Bureau de tabac & R-kioski \\
\hline \multirow{2}{*}{ Tournure sans verbe } & $\mathrm{n}$ & 44 & 49 \\
\cline { 2 - 4 } & $\boldsymbol{\%}$ & $\mathbf{4 9 , 4}$ & $\mathbf{5 9 , 0}$ \\
\hline \multirow{2}{*}{ Proposition impérative } & $\mathrm{n}$ & 2 & 1 \\
\cline { 2 - 4 } & $\boldsymbol{\%}$ & $\mathbf{2 , 2}$ & $\mathbf{1 , 2}$ \\
\hline \multirow{2}{*}{ Proposition affirmative } & $\mathrm{n}$ & 31 & 19 \\
\cline { 2 - 4 } & $\boldsymbol{\%}$ & $\mathbf{3 4 , 8}$ & $\mathbf{2 2 , 9}$ \\
\hline Total & $\mathrm{n}$ & 12 & 14 \\
\cline { 2 - 4 } & $\boldsymbol{\%}$ & $\mathbf{1 3 , 5}$ & $\mathbf{1 6 , 9}$ \\
\hline
\end{tabular}

Tableau 7. Distribution des requêtes verbales selon le type de proposition 
Ce tableau illustre les principales différences: le finnois utilise plus de tournures sans verbe que le français et le français utilise plus de propositions affirmatives que le finnois.

Quelles sont les raisons qui expliquent ces choix stratégiques ? Il est clair qu'il existe une différence entre les produits qui doivent être demandés et les produits qui se trouvent en libre-service : ces derniers ne nécessitent pas de formulation verbale. Kerbrat-Orecchioni (2001b : 11) affirme que les questions sont utilisées lorsque le client ne voit pas le produit immédiatement tandis que « je vais prendre $\mathrm{X}$ » est utilisé «quand le produit est manifestement ou vraisemblablement disponible ». Les affirmations d'un désir fonctionnent comme des formulations « passe-partout» (ibid.). La position et la disponibilité des produits sont donc des aspects qui influencent le choix de la stratégie. De plus, Sorjonen, Raevaara \& Lappalainen (2009) ont montré que l'âge et le sexe du client avaient un effet sur la stratégie utilisée dans les $R$-kioski : les femmes et les personnes âgées emploient plus de propositions que les hommes et les personnes jeunes.

Sorjonen \& Raevaara (2014) ont étudié les requêtes de produits de tabac et la variation entre un syntagme nominal et une proposition dans les R-kioski. Leur étude montre que le choix entre ces deux stratégies est lié à la multimodalité des interactions : les clients utilisent une formulation longue (une proposition) quand ils sont en train de s'approcher du comptoir tandis qu'une formulation courte (un syntagme nominal) est choisie quand le client se trouve déjà au stade transactionnel. Dans cette étude finlandaise, le choix de la formulation montre donc comment le client interprète la distance entre lui et le comptoir (Sorjonen \& Raevaara, $2014: 263$ ).

\subsection{Distribution de la perspective}

Nous avons également classifié les requêtes verbales des deux corpus étudiés selon leur perspective. En voici les résultats :

\begin{tabular}{|l|c|c|c|}
\hline Perspective & & Bureau de tabac & R-kioski \\
\hline \multirow{2}{*}{ Orientée vers le locuteur } & $\mathrm{n}$ & 29 & 17 \\
\cline { 2 - 4 } & $\mathbf{\%}$ & $\mathbf{3 2 , 6}$ & $\mathbf{2 0 , 5}$ \\
\hline \multirow{2}{*}{ Impersonné vers l'auditeur } & $\mathrm{n}$ & 11 & 6 \\
\cline { 2 - 4 } & $\boldsymbol{\%}$ & $\mathbf{1 2 , 4}$ & $\mathbf{7 , 2}$ \\
\hline \multirow{2}{*}{ Inclusive } & $\mathrm{n}$ & 49 & 60 \\
\cline { 2 - 4 } & $\boldsymbol{\%}$ & $\mathbf{5 5 , 1}$ & $\mathbf{7 2 , 3}$ \\
\hline Total & $\mathrm{n}$ & 0 & 0 \\
\cline { 2 - 4 } & $\mathbf{\%}$ & $\mathbf{0 , 0}$ & 83 \\
\hline
\end{tabular}

Tableau 8. Distribution des requêtes verbales selon la perspective

La perspective impersonnelle est la perspective la plus employée dans les deux corpus mais elle est clairement plus courante en finnois. Cela est sans doute lié au choix de la stratégie de la requête : comme nous venons de le mentionner, les tournures sans verbe sont impérativement impersonnelles et elles forment la majeure partie des requêtes finnoises. Dans le corpus français, les affirmations sont presque toujours orientées vers le locuteur («je voudrais $X »$, «je prends $X$ » etc.) à l'exception de quelques cas spéciaux (p. ex. «alors c'est télé poche qu'elle m'a dit», «c'est pour la vignette auto »). En finnois, par contre, il y a plus de variation : la perspective des affirmations est également le plus souvent orientée vers le locuteur («otan $X »($ je prends $\mathrm{X})$, «ostaisin $X »(\mathrm{j}$ 'acheterais $\mathrm{X})$ ), mais la perspective impersonnelle n'est pas rare non plus («laitetaan $X »($ on met $\mathrm{X}$ ), «pistetään $X »($ on met $\mathrm{X}$ ), «otetaan $X$ » (on prend $\mathrm{X})$ ). En effet, en finnois, il y a plus de façons de formuler des requêtes impersonnelles qu'en français : en français, quelques cas exceptés, toutes les requêtes impersonnelles sont des tournures sans verbe tandis qu'en finnois, on trouve des requêtes comme «otetaan toi puol ravi ( on prend un pari hippique). Il est naturel de demander «onko multifiltteri sikareita» en finnois, mais son équivalent direct en français « est-ce qu'il y a des cigares multifiltres » serait bizarre dans ce contexte, ce qui montre la différence 
entre les deux langues-cultures. En effet, d'après Yli-Vakkuri (2005), le finnois évite en général de mentionner l'interlocuteur et préfère les stratégies impersonnelles.

\subsection{Distribution des modificateurs internes}

Comme nous l'avons déjà indiqué ci-dessus (voir 3.3.3.), les modificateurs de requête diffèrent en principe entre le français et le finnois. Qu'en est-il dans le contexte du bureau de tabac et du $R$-kioski ? Pour répondre à cette question, nous avons repéré toutes les requêtes qui contiennent un prédicat qui puisse être modifié, c'est-à-dire les affirmations et les interrogations, pour y analyser les modificateurs syntaxiques. Les éléments lexicaux ont été examinés dans toutes les requêtes.

\begin{tabular}{|c|c|c|c|c|}
\hline & & & Bureau de tabac & R-kioski \\
\hline \multirow{9}{*}{$\begin{array}{l}\text { Modification } \\
\text { morpho- } \\
\text { syntaxique }\end{array}$} & \multirow[t]{2}{*}{ Conditionnel } & $\mathrm{n}$ & 15 & 7 \\
\hline & & $\%$ & 34,9 & 21,2 \\
\hline & \multirow[t]{2}{*}{ Imparfait } & $\mathrm{n}$ & 2 & 0 \\
\hline & & $\%$ & 4,7 & $\mathbf{0 , 0}$ \\
\hline & \multirow[t]{2}{*}{ Négation } & $\mathrm{n}$ & 2 & 1 \\
\hline & & $\%$ & 4,7 & 3,0 \\
\hline & \multirow{2}{*}{$\begin{array}{l}\text { Pas de modification } \\
\text { morpho-syntaxique }\end{array}$} & $\mathrm{n}$ & 23 & 25 \\
\hline & & $\%$ & 55,8 & 75,8 \\
\hline & Total & $\mathrm{n}$ & 43 & 33 \\
\hline \multirow{7}{*}{$\begin{array}{l}\text { Modification } \\
\text { lexicale }\end{array}$} & \multirow[t]{2}{*}{ Marqueur de politesse } & $\mathrm{n}$ & 26 & 1 \\
\hline & & $\%$ & 29,2 & 1,2 \\
\hline & \multirow[t]{2}{*}{ Minimisation } & $\mathrm{n}$ & 3 & 0 \\
\hline & & $\%$ & 3,4 & $\mathbf{0 , 0}$ \\
\hline & \multirow{2}{*}{$\begin{array}{l}\text { Pas de modification } \\
\text { lexicale }\end{array}$} & $\mathrm{n}$ & 60 & 82 \\
\hline & & $\%$ & 67,4 & 98,8 \\
\hline & Total & $\mathrm{n}$ & 89 & 83 \\
\hline
\end{tabular}

\section{Tableau 9. Distribution de la modification interne}

Le tableau 9 montre les différences entre les corpus : le français utilise plus de modificateurs morphosyntaxiques (le conditionnel, l'imparfait et la négation) et lexicaux (les marqueurs de politesse, la minimisation) comparé au finnois. Dans le corpus finnois, la plupart des requêtes ne sont aucunement modifiées: $76 \%$ des requêtes affirmatives et interrogatives ne sont pas modifiées morphosyntaxiquement et on ne trouve qu'une seule instance de modification lexicale dans tout le corpus. En français, par contre, presque la moitié (44\%) des requêtes affirmatives et interrogatives sont modifiées morpho-syntaxiquement, le conditionnel étant le cas le plus courant. Dans le contexte du bureau de tabac, la modification lexicale n'est pourtant pas courante en français non plus : on trouve le marqueur de politesse $s$ 'il vous plaît seulement dans $29 \%$ des requêtes verbales. Il accompagne le plus souvent une tournure sans verbe (p. ex. « une cartouche de gauloises filtres s'il vous plaît») ou l'affirmation d'un désir (p. ex. «je voudrais deux paquets de gauloises blondes s’il vous plaît »).

Est-ce qu'il y a des facteurs qui expliquent la présence ou l'absence des modificateurs internes ? Sorjonen, Raevaara \& Lappalainen (2009) affirment que l'emploi du conditionnel est lié à la place du produit dans le magasin : en finnois, le mode conditionnel («mä ottaisin » (je prendrais)) est utilisé le plus souvent quand le produit que le client est en train d'acheter doit être demandé au vendeur, tandis que le mode indicatif («mä otan (je prends)) est employé quand le client a pris lui-même le produit désiré dans le rayon. Cette observation montre, selon elles, que l'emploi ou l'absence du conditionnel n'est pas lié à la politesse mais à l'organisation physique de la situation de service. Kerbrat-Orecchioni (2005b : 36), quant à elle, voit le conditionnel comme un élément qui adoucit les directives françaises en général. Dans le même article, elle affirme que le marqueur de politesse s'il vous plaît accompagnerait «invariablement» les tournures sans verbe dans les petits commerces, mais dans une publication 
postérieure (Kerbrat-Orecchioni, 2008), elle avoue que cet élément n'apparaît pas systématiquement. Le corpus étudié ici montre que $s$ 'il vous plaît n'est pas un modificateur qui accompagnerait obligatoirement les tournures sans verbe : des 44 requêtes formulées sans verbe 19 seulement (soit $43 \%$ ) contiennent ce marqueur de politesse - c'est-à-dire que la majorité $(\mathrm{n}=25$, soit $57 \%)$ des tournures sans verbe ne sont pas atténuées à l'aide de ce modificateur lexical. Cela nous semble surprenant étant donné que $s$ 'il vous plâ̂t « concilie les exigences de la clarté, de la rapidité (économie maximale de l'information), et de la politesse » (Kerbrat-Orecchioni, $2008:$ 117).

\section{Conclusion}

Dans cet article, nous avons traité les différentes manières de réaliser des requêtes dans un bureau de tabac français et dans son équivalent finlandais, $R$-kioski. Dans les deux corpus, les requêtes peuvent être produites aussi bien verbalement que non-verbalement. En analysant les stratégies de requête verbale, nous avons vu que l'emploi d'une tournure sans verbe est la stratégie la plus utilisée dans les deux corpus, mais qu'elle est plus fréquente en finnois qu'en français. Une autre différence importante concerne l'affirmation d'un désir comme stratégie, puisqu'elle n'apparaît pas dans le corpus finnois tandis qu'elle est assez courante dans le corpus français. En ce qui concerne la perspective de requête, les deux langues préfèrent la perspective impersonnelle. Il est pourtant important de noter que la perspective est fortement liée à la stratégie de requête. C'est donc probablement à cause du taux élevé de l'emploi d'une tournure sans verbe que la perspective impersonnelle est plus courante en finnois qu'en français. Quant à la modification interne des requêtes, le français utilise considérablement plus de modificateurs morphosyntaxiques et lexicaux que le finnois. Il emploie notamment assez souvent le marqueur de politesse $s$ ' $i l$ vous plaît tandis que l'équivalent finnois le plus proche, kiitos, ne s'emploie guère.

En général, les stratégies de requête sont donc assez similaires dans le bureau de tabac et dans le R-kioski. Dans un tel contexte, les stratégies efficaces et courtes sont préférées. Pourtant, la distribution des stratégies ainsi que de la perspective diffèrent entre les deux corpus. La plus grande différence se trouve dans la modification interne des requêtes : on pourrait dire que dans un bureau de tabac français, la requête typique est « je voudrais $\mathrm{X} »$ ou « $\mathrm{X}$ (s'il vous plaît) », tandis que dans un $R$-kioski finlandais il suffit de dire «mä otan $X »($ je prends $\mathrm{X})$ ou tout simplement « $X »$.

Nous rappelons que les deux corpus ne datent pas de la même période et qu'ils ne sont pas tout à fait récents. Il ne faut donc pas tirer de conclusions et les appliquer à des situations de service d'aujourd'hui sans une certaine réserve. En outre, nous n'avons étudié qu'un seul site en France et en Finlande - nous nous défendons donc de prétendre que ces résultats représentent la situation globale des deux languescultures en question. De plus, nous voulons insister sur l'effet du contexte : ces résultats montrent seulement comment les requêtes sont produites dans un bureau de tabac. La quotidienneté, la place et le type des produits ont une influence importante sur les réalisations des requêtes. Cela explique pourquoi les requêtes ne sont pas produites dans un bureau de tabac de la même manière que dans un magasin de vêtements, par exemple. Malgré ces réserves, nous croyons avoir pu montrer certaines différences générales entre les deux langues. Il serait du reste intéressant de comparer les résultas avec des données plus récentes pour voir d'éventuelles différences et d'élargir la comparaison des requêtes françaises et finnoises à d'autres contextes pour avoir une idée plus large des différences entre ces langues-cultures.

\section{Conventions de transcription}

Les conventions de transcription suivent celles d'ICOR 9 .

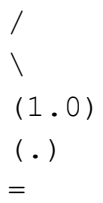

$(1.0)$ intonation montante intonation descendante pause d'une seconde micro-pause enhaînement immédiat 


\begin{tabular}{|c|c|}
\hline $\begin{array}{r}\operatorname{me}[\operatorname{rci}] \\
{[\operatorname{merci}]}\end{array}$ & chevauchement \\
\hline$(($ entre) ) & action \\
\hline p’tit & élision non-standard \\
\hline me:rci & allongement \\
\hline (je veux) & segment incertain \\
\hline BONjour & saillance perceptuelle \\
\hline je crois ${ }^{\circ}$ & baisse du volume de la voix \\
\hline$(($ rire $))$ & production vocale \\
\hline$<(($ lentement $))$ alors $>$ & description \\
\hline 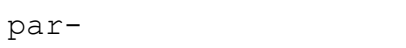 & troncation \\
\hline. $\mathrm{h}$ & aspiration \\
\hline
\end{tabular}

\section{Références bibliographiques}

Aijmer, K. (1996). Conversational Routines in English. Convention and Creativity. Studies in Language and Linguistics. London : Longman.

Blum-Kulka, S. (1989). Playing It Safe: The Role of Conventionality in Indirectness. In Blum-Kulka, S., House, J. \& Kasper, G. (éds), Cross-Cultural Pragmatics: Requests and Apologies, Norwood : Ablex, 37-70.

Blum-Kulka, S. \& House, J. (1989). Cross-Cultural and Situational Variation in Requesting Behavior. In BlumKulka, S., House, J. \& Kasper, G. (éds), Cross-Cultural Pragmatics: Requests and Apologies, Norwood : Ablex, $123-154$.

Blum-Kulka, S., House, J. \& Kasper, G. (éds) (1989). Cross-Cultural Pragmatics: Requests and Apologies. Advances in discourse processes 31. Norwood: Ablex.

Brown, P. \& Levinson, S. (1987). Politeness. Some universals in language usage. Cambridge : Cambridge University Press.

Drew, P. \& Couper-Kuhlen, E. (2014). Requesting - from speech act to recruitment. In Drew, P. \& Couper-Kuhlen, E. (éds), Requesting in Social Interaction, Amsterdam : John Benjamins Publishing Company, 1-34.

Drew, P. \& Couper-Kuhlen, E. (éds) (2014). Requesting in Social Interaction. Studies in Social Interaction 26. Amsterdam : John Benjamins Publishing Company.

Fox, B. (2015). On the notion of pre-requests. Discourse Studies, 17 (1), 41-63.

Haakana, M. \& Sorjonen, M.-L. (2009). Miljoonia anomassa ja nostamassa. Leikittelyä kioskin asioinneissa. In Lappalainen, H. \& Raevaara, L. (éds), Kieli kioskilla. Tutkimuksia kioskiasioinnin rutiineista, Helsinki : Suomalaisen Kirjallisuuden Seura, 201-230.

Hmed, N. (2008). Analyse comparative d'interactions dans des petits commerces français, tunisien et francomaghrébien. In Kerbrat-Orecchioni, C. \& Traverso, V. (éds), Les interactions en site commercial. Invariants et variations, Lyon : Ens Éditions, 253-276.

Kerbrat-Orecchioni, C. (2001a). Les actes de langage dans le discours. Paris : Nathan.

Kerbrat-Orecchioni, C. (2001b). Je voudrais un p'tit bifteck. Les Carnets du Cediscor, 7 [en ligne, consulté le 10 mars 2016]. http://cediscor.revues.org/307

Kerbrat-Orecchioni, C. (2005a). Le discours en interaction. Paris : Armand Colin.

Kerbrat-Orecchioni, C. (2005b). Politeness in France: How To Buy Bread Politely. In Hickey, L. \& Stewart, M. (éds) Politeness in Europe. Clevedon : Multilingual Matters, 29-44.

Kerbrat-Orecchioni, C. (2008). Les interactions en site commercial : des interactions « polies ». In KerbratOrecchioni, C. \& Traverso, V. (éds), Les interactions en site commercial. Invariants et variations, Lyon : Ens Éditions, 105-137.

Kerbrat-Orecchioni, C. \& Traverso, V. (éds) (2008). Les interactions en site commercial. Invariants et variations. Lyon : Ens Éditions. 
Lappalainen, H. (2009a). Tervehtiminen ja tervehtimättä jättäminen. Ollaanko kioskilla epäkohteliaita? In Lappalainen, H. \& Raevaara, L. (éds), Kieli kioskilla. Tutkimuksia kioskiasioinnin rutiineista, Helsinki : Suomalaisen Kirjallisuuden Seura, 32-55.

Lappalainen, H. (2009b). Hei, moi ja huomenta. Tervehdykset kioskilla. In Lappalainen, H. \& Raevaara, L. (éds), Kieli kioskilla. Tutkimuksia kioskiasioinnin rutiineista, Helsinki : Suomalaisen Kirjallisuuden Seura, 56-89.

Lappalainen, H. \& Raevaara, L. (éds) (2009). Kieli kioskilla. Tutkimuksia kioskiasioinnin rutiineista. Kotimaisten kielten tutkimuskeskuksen julkaisuja. Helsinki : Suomalaisen Kirjallisuuden Seura.

Levinson, S. C. (1983). Pragmatics. Cambridge Textbooks in Linguistics. Cambridge : Cambridge University Press.

Muikku-Werner, P. (1997). "Jättä minut rauhaan, hävi heti”. Suomalaisten ja suomenoppijoiden pyynnöt ja anteeksipyynnöt. Jyväskylä : Jyväskylän yliopisto, soveltavan kielentutkimuksen keskus.

Peterson, E. (2008). Pressure to please: the case of English and politeness in Finland. In Vergaro, C. (éd.), Dynamics of language contact in the twenty first century, 2 (1), Perugia, Italy : Guerra Edizioni, 161-177.

Peterson, E. (2010). Perspective and politeness in Finnish requests. Pragmatics, 20 (3), 401-423.

Peterson, E. \& Vaattovaara, J. (2014). Kiitos and pliis: The relationship of native and borrowed politeness markers in Finnish. Journal of Politeness Research, 10 (2), 247-269.

Rossi, G. (2014). When do people not use language to make requests? In Drew, P. \& Couper-Kuhlen, E. (éds), Requesting in Social Interaction, Amsterdam : John Benjamins Publishing Company, 303-334.

Schegloff, E. A. (2007). Sequence Organization in Interaction. A Primer in Conversation Analysis, Volume 1. Cambridge : Cambridge University Press.

Sorjonen, M.-L. \& Raevaara, L. (2014). On the grammatical form of requests at the convenience store. Requesting as embodied action. In Drew, P. \& Couper-Kuhlen, E. (éds), Requesting in Social Interaction, Amsterdam : John Benjamins Publishing Company, 243-268.

Sorjonen, M.-L., Raevaara, L. \& Lappalainen, H. (2009). Mä otan tän. Käynnin syyn esittämisen tavat kioskilla. In Lappalainen, H. \& Raevaara, L. (éds), Kieli kioskilla: tutkimuksia kioskiasioinnin rutiineista, Helsinki : Suomalaisen Kirjallisuuden Seura, 90-119.

Traverso, V. (2006). Aspects of polite behavior in French and Syrian service encounters: A data-based comparative study. Journal of Politeness Research, 2, 105-122.

Van Mulken, M. (1996). Politeness markers in French and Dutch requests. Language Sciences, 18 (3-4), 689-702.

VISK = Hakulinen, A., Vilkuna, M., Korhonen, R., Koivisto, V., Heinonen, T. R. \& Alho, I. (2004). Iso suomen kielioppi [Grande grammaire du finnois]. Version électronique, 2008 [consulté le 10 mars 2016]. Helsinki : Suomalaisen Kirjallisuuden Seura. http://scripta.kotus.fi/visk

Yli-Vakkuri, V. (2005). Politeness in Finland: Evasion at All Costs. In Hickey, L. \& Stewart, M. (éds), Politeness in Europe, Clevedon : Multilingual Matters, 189-202.

\footnotetext{
${ }^{1}$ Nous avons fait quelques sélections dans les deux corpus pour qu'ils soient comparables. Par exemple, nous avons exclu du corpus finnois les interactions où les clients touchaient des gains parce que la nature de ces interactions est différente : le client ne paie pas pour un objet ou un service, mais c'est la buraliste qui rembourse le client. Nous ne traiterons pas non plus les situations où la buraliste sert un couple.

${ }^{2}$ Nous utilisons ces corpus pour profiter de données qui ont déjà été recueillies et étudiées par d'autres chercheurs (pour le corpus français, voir Kerbrat-Orecchioni \& Traverso 2008 et pour le corpus finnois, voir Lappalainen \& Raevaara 2009). Nous remercions l'équipe de recherche ICAR (Université Lyon 2) et le centre de langues nationales de Finlande (Kotus) de nous avoir donné accès à ces corpus.

${ }^{3}$ Sur la salutation dans les R-kioski finlandais, voir Lappalainen (2009a, 2009b). Sur la salutation dans les petits commerces français, voir Kerbrat-Orecchioni (2008). Mentionnons que dans les deux langues, le premier tour de salutation est le plus souvent prononcé par le buraliste et qu'en finnois, la variation des différentes formulations est beaucoup plus grande qu'en français (hei [cas le plus courant], moi, päivää, terve, huomenta etc. vs. bonjour [cas le plus courant], bonsoir, salut).
} 


\begin{abstract}
${ }^{4}$ Dans les transcriptions françaises, BEA correspond à la buraliste. Les clients sont numérotés : CL1, CL2, etc. Nous utilisons les transcriptions faites par l'équipe ICAR. Cependant, nous avons ajouté des notes liées aux actions, marquées entre parenthèses doubles, parce que les transcriptions originales en contiennent très peu.

${ }^{5}$ Dans les transcriptions finnoises, A correspond au client (asiakas en finnois) et M à la buraliste (myyjä en finnois). Les clients ne sont pas numérotés, mais chaque situation d'interaction a son propre code. Nous utilisons les transcriptions faites par l'équipe qui a rassemblé les données, mais nous les avons simplifiées un peu. Nous avons également fait quelques modifications pour que la transcription corresponde à la convention française. Les traductions des tours sont marquées en gris et en italiques. En traduisant, nous n'avons pas fait d'adaptations pragmatiques en français : par exemple, quand la buraliste finlandaise tutoie ses clients (ce qui est tout à fait normal dans le contexte finnois), nous utilisons le tutoiement également dans la traduction française. C'est également le cas pour les salutations : «moi », qui est une salutation moins formelle, a été traduit par « salut » en français.
\end{abstract}

${ }^{6}$ Dans les requêtes du type «X, s'il vous plaît », il y a bien évidemment le verbe plaire, mais comme il apparaît dans une formule figée, nous désignons ces requêtes par « tournures sans verbe ».

7 Selon la Grande grammaire du finnois VISK ( $\$ 1654$ ), la voix passive dans les directifs («mennään» (on va), «yritetään » (on essaie) etc.) peut être inclusive ou exclusive selon le verbe et le contexte où elle apparaît. Dans le contexte du $R$-kioski, il nous semble que le référent n'est pas tout à fait clair (est-ce que «laitetaan» (on met) veut dire que c'est la buraliste ou le client ou les deux qui agissent ?). Nous considérons donc que l'emploi du passif doit faire partie de la perspective impersonnelle.

${ }^{8}$ Remarquons cependant que Muikku-Werner a utilisé un Discourse Completion Test comme méthode et qu'elle n'a pas étudié les requêtes dans des situations de service.

${ }^{9}$ http://icar.univ-lyon2.fr/projets/corinte/documents/2013_Conv_ICOR_250313.pdf 